\title{
Multi-spark modeling of very fast transient overvoltages for the purposes of developing HV and UHV gas-insulated switchgear and of conducting insulation co-ordination studies
}

\author{
M. SZEWCZYK* \\ ABB Corporate Research Center, 31-038 Krakow, Starowislna 13A, Poland
}

\begin{abstract}
Very fast transient overvoltages (VFTO) originate from steep voltage breakdowns in $\mathrm{SF}_{6}$ gas that are inherent to operation of any switching device of the gas-insulated switchgear (GIS) type. For power stations with voltage ratings exceeding $500 \mathrm{kV}$, the ratio between equipment rated- and withstand-voltage levels becomes relatively low, which causes the VFTO peak values to reach the component's insulation withstand-voltage levels, thus becoming a design factor for high- and ultra-high voltage GIS. While well-established approach to VFTO analyses involves only single VFTO events (the so-called single-spark approach), there is often the need to analyze the entire VFTO generation process, for which the multi-spark approach to VFTO modeling is to be employed. The multi-spark approach allows one to evaluate the VFTO impact on the GIS disconnector design along with the impact of the VFTO on selection and dimensioning of the VFTO damping solutions. As the multi-spark approach to VFTO modeling is now being increasingly used in UHV GIS developments as well as for the insulation co-ordination studies of power stations, the present paper is motivated by the need to report on the VFTO multi-spark modeling approach and to lay a common ground for development works that are supported extensively with VFTO simulations. The paper presents physical assumptions and modeling concepts that are in use in such modeling works. Development of the multi-spark GIS disconnector model for VFTO simulations is presented, followed by an overview of examples of the model application for the GIS development works and for insulation co-ordination studies.
\end{abstract}

Key words: very fast transient overvoltages (VFTO), gas-insulated switchgear (GIS), disconnector switch (DS), modeling, simulations, transients, switching, mitigation, breakdown voltage (BDV) characteristics.

\section{Introduction}

Although pioneering work on very fast transient (VFT) overvoltages (VFTO) in gas-insulated switchgear (GIS) dates back to the 1980s [1, 2], the importance of accurate VFTO modeling has been renewed with recent developments in high- and, most importantly, ultra-high voltage (UHV) GIS. These developments, intended mostly for super-grids of UHVAC transmission systems in China and India, have reached rated voltage levels as high as $1100 \mathrm{kV} \mathrm{AC}$.

As the VFTO originate from flashovers in $\mathrm{SF}_{6}$ gas, they are associated with operation of GIS switching devices, primarily disconnectors, and as such cannot be avoided in any GIS. Specifically for UHV GIS, the VFTO have become a design factor, which was due to the decreased ratio between rated voltage and the insulation co-ordination withstand-voltage levels of the GIS components and substations (as per IEC Std. [3]). Consequently, VFTO analyses are now being increasingly employed for design work and to support type testing of (primarily) GIS disconnectors $[4,5]$. Furthermore, the VFTO analyses results are often used to support a decision on application, selection and dimensioning of the VFTO damping solutions $[6,7]$, which in some cases are required to maintain the VFTO peak values at an

*e-mail: marcin.szewczyk@pl.abb.com

Manuscript submitted 2017-03-21, revised 2017-08-09, initially accepted for publication 2017-08-12, published in December 2017. acceptable level. Testing of GIS components, when supported with accurate and verified models, allows for a cost-effective design process and avoiding unwanted flashovers between live and earthed elements during tests and normal operation.

To explore the VFTO associated with the operation of the UHV GIS disconnectors, the State Grid Corporation of China (SGCC) has recently established two full-scale test set-ups at the $1100 \mathrm{kV}$ station in Wuhan (China), and conducted measuring campaigns with the total number of disconnector operations of approximately 2000 [8]. Several papers have been published based on this work, addressing different aspects of VFTO measurements and simulations. As an example, in [9] the impact of the disconnectors moving contact speed on the variety of VFTO parameters is analyzed.

Also in the previous works conducted by ABB, the VFTO have been investigated to support design work of the $1100 \mathrm{kV}$ disconnector $[10,11]$ and to analyze the impact of the VFTO on selection of the VFTO damping solutions [6]. These works utilized the so-called multi-spark approach $[4,12]$ for modeling the entire process of disconnector operation and to analyze distributions of the VFTO, along with other parameters, such as trapped charge voltage distributions, arcing time and number of sparks - and the mutual effect of these qualities and the GIS disconnector design.

The renewed interest in analyzing VFTO in HV and UHV GIS has led to the need for embedding detailed and accurate modeling and simulations of VFTO in the development works of the GIS components and installations, as well as in the insu- 
lation co-ordination studies of the GIS-AIS power stations. It has also motivated this paper, which is to present a comprehensive approach to multi-spark VFTO modeling in GIS. The paper provides a description of physical assumptions required for accurate modeling of VFTO, jointly with the description of the development of the GIS multi-spark disconnector simulation model. Critical parameters of the disconnector model are also described.

The paper is organized as follows: Section I gives background information and motivation for using the multi-spark approach to VFTO modeling in GIS. Section II outlines the process of VFTO generation and gives rationales on why this process is analyzed in the context of GIS disconnectors operation. Section III includes a description of key physical assumptions relevant for VFTO modeling, and Section IV focuses on modeling the disconnector breakdown voltage characteristics. Section V describes the development of the multi-spark GIS disconnector model, which includes the GIS disconnector itself as well as other components of the GIS substation or test set-up. Section VI presents examples of simulation results obtained with the multi-spark VFTO modeling approach presented in this paper. The results are then presented for simplified GIS set-ups, followed by an overview of example simulations conducted for full-scale $1100 \mathrm{kV}$ GIS test set-ups. The VFTO mitigation techniques are also outlined to illustrate the importance of multispark VFTO analyses for supporting a decision on application, selection and dimensioning of the VFTO damping solutions. Finally, Section VII offers conclusions.

\section{VFTO generation process}

VFTO generation takes place during operation of any GIS switching device, however the operation of the GIS disconnectors raises most concerns. This is primarily due to the relatively slow operation process of the disconnectors, = as compared to other switching devices. This leads to the highest number of associated breakdown flashovers occurring in the disconnector contact system during the disconnector opening and closing operations.

Figure 1 shows an example of the GIS pole layout, as piloted in the Jingmen $1100 \mathrm{kV}$ station in China [10], where the "breaker-and-a-half" configuration of the GIS pole was used.

The VFTO generation process is determined by voltage conditions on the source- and on the load-side of the discon-

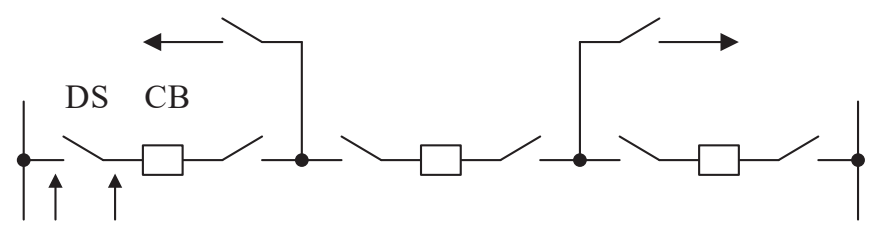

$\mathrm{u}_{\mathrm{S}} \quad \mathrm{u}_{\mathrm{L}}$

Fig. 1. "Breaker-and-a-half" configuration of a GIS pole as piloted in the Jingmen $1100 \mathrm{kV}$ AIS-GIS hybrid substation [10]: DS - disconnector operated, CB - circuit breaker; $u_{\mathrm{S}}$ - source-side $50 / 60 \mathrm{~Hz}$ voltage, $u_{\mathrm{L}}$ - load-side voltage on the GIS busbar between disconnector DS and circuit breaker CB nector contact system, by the voltage breakdown characteristics in the disconnector contact gap (the so-called sparks), and by the parameters characterizing the GIS components that the disconnector is connected to. The voltage conditions primarily determine the instances of spark ignitions, and thus, jointly with the parameters of the other GIS components that are connected to the disconnector, determine the peak values of the resultant VFTO. The breakdown characteristics of the disconnector contact system primarily determine the high frequency nature of the VFTO waveforms.

VFTO peak values can reach up to 2.8 p.u. [10] (where 1 p.u. $=V_{\mathrm{r}} \cdot \sqrt{2} / \sqrt{3}, V_{\mathrm{r}}-$ rated voltage $)$. The frequencies of the VFTO components go up to $100 \mathrm{MHz}$ [13], which are the highest frequencies observed among all types of power system transient studies. For HV and UHV rated voltages, the associated current waveforms are characterized by peak values reaching $\mathrm{kA}[1]$.

During the disconnector operation, the associated circuit breaker remains opened (which is relevant for the substation operation and reflected in the type test layouts and standardized testing procedures). The unloaded, capacitive GIS busbar between the disconnector and the circuit breaker (see Fig. 1) is thus being disconnected from and then connected to the sourceside voltage $u_{\mathrm{S}}$ by the disconnector switch opening and closing operation.

\subsection{Voltage conditions and breakdown flashovers (sparks).} The VFTO generation process involves several stages. As the disconnector moving contact separates itself from the fixed contact (for the opening operation), or approaches the fixed contact (for the closing operation), the electric field gradient between the two contacts rises. This is due to the fact (see Fig. 1 and Fig. 2) that the source-side voltage $u_{\mathrm{S}}$ alters with the system frequency $(50 / 60 \mathrm{~Hz})$ while the load-side voltage $u_{\mathrm{L}}$ remains constant on the capacitive and unloaded busbar between the disconnector and the circuit breaker (neglecting charge decay of the

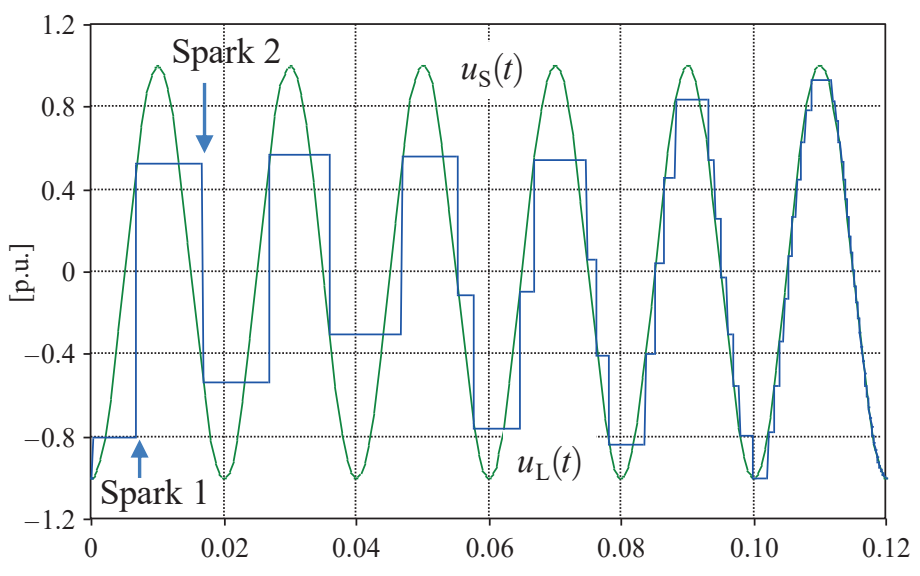

[s]

Fig. 2. Illustrative VFT waveform (with typical step-wise pattern) of $u_{\mathrm{S}}$ - source-side voltage (green), and $u_{\mathrm{L}}$ - load-side voltage (blue), as indicated in Fig. 1; voltage breakdown events (Spark 1 and Spark 2) are indicated as referenced in the text 
trapped charge that is occurring on the load-side busbar). When the voltage across the disconnector contacts $\left(u_{\mathrm{L}}-u_{\mathrm{S}}\right)$ exceeds the instantaneous breakdown voltage value (which depends on the actual contact gap length, on the design of the disconnector contact system and on the $\mathrm{SF}_{6}$ gas characteristics and pressure), the first breakdown flashover (spark) occurs. The arc current associated with the breakdown flashover causes the capacitive load to be charged to the source-side voltage $u_{\mathrm{S}}$ instantaneous value, so that $u_{\mathrm{L}}$ equals $u_{\mathrm{S}}$. This causes the arcing current across the contacts to drop, and thus the spark is extinguished, which then causes transient recovery voltage $\left(T R V=u_{\mathrm{L}}-u_{\mathrm{S}}\right)$ to build up. The subsequent spark occurs when the $T R V$ across the contacts exceeds the breakdown voltage again. As the source-side voltage $u_{\mathrm{S}}$ changes with $50 / 60 \mathrm{~Hz}$ frequency while the load-side voltage $u_{\mathrm{L}}$ remains constant (neglecting charge decay), the process continues until the breakdown voltage rises enough to withstand the $T R V$ occurring across the contacts (for the opening operation) or until the breakdown voltage reaches zero (for the closing operation).

According to the process described, load-side voltage $u_{\mathrm{L}}$ follows source-side voltage in a step-wise manner, as shown in Fig. 2 for illustrative example of the disconnector closing operation. The process shown in Fig. 2 is presented for illustration purposes only, i.e. the breakdown voltage characteristics used to obtain the $u_{\mathrm{L}}$ waveform are not related to any actual GIS disconnector design.

The so-called single-spark modeling approach $[14,15]$ involves modeling a single spark only from the entire process depicted in Fig. 2. In the multi-spark modeling approach [4], voltage conditions are simulated for the entire process of the GIS disconnector operation, and then all of the VFTO events are represented in the model as occurring for specific times of the spark ignitions.

2.2. Travelling waves superimposition. Each of the sparks occurring in $\mathrm{SF}_{6}$ gas in the contact gap during the process of opening and closing operation of the GIS disconnector, produces a steep voltage rise on the unloaded busbar between the disconnector and the circuit breaker. As the rise time of the voltage breakdown is significantly smaller than that of the electromagnetic wave transit time through the GIS components, the travelling waves originate from every spark and then propagate through the GIS. The wave peak value is governed by the voltage conditions $\left(u_{\mathrm{S}}\right.$ and $u_{\mathrm{L}}$, see e.g. Fig. 2$)$ at the time instance of spark ignition. The wave steepness is governed by the voltage breakdown characteristics in $\mathrm{SF}_{6}$ gas.

The travelling wave propagates both inside and outside of the GIS. The inside part constitutes VFTO, while the outside part constitutes the so-called transient enclosure voltages (TEV) [16]. Initially, the wave also crosses the disconnector gap being short-circuited by the electric arc of the spark. When the arc is eventually extinguished, the wave reflects also from the opened disconnector contact gap as it does at every discontinuity point of the surge impedance of the GIS components and open ends. The VFTO is finally formed by superimposition of the travelling waves that emerge from multiple reflections and transmissions on the surge impedance discontinuities.

\section{Physical assumptions of VFTO modeling}

This section presents a description of physical phenomena and assumptions that are used in modeling of the breakdown flashovers in the GIS disconnector contact system, and in modeling of the so-called trapped charge voltage occurring on the GIS busbar between the disconnector and the associated circuit breaker.

3.1. Breakdown time. Breakdown flashovers occur in the disconnector contact gap when the voltage across the disconnector contact system exceeds the instantaneous value of breakdown voltage. A conducing spark channel is then established between the contacts within the so-called breakdown time $t_{\mathrm{B}}$, which is in the order of just a few nanoseconds. During this time, the spark resistance changes from a very large value (representing the contact gap insolating characteristics) to a relatively small value (representing the electric arc resistance).

The time duration $t_{\mathrm{B}}$ of the spark ignition or extinction process in a gas is calculated by means of the Toepler equation [13]:

$$
t_{\mathrm{B}}=13.3 \frac{k_{T}}{E_{0} \cdot \eta \cdot p},
$$

where: $k_{T}=40 \div 70 \mathrm{kV} \cdot \mathrm{ns} / \mathrm{cm}$ is the Toepler coefficient for $\mathrm{SF}_{6}$ gas at the pressure of $0.1 \div 2 \mathrm{MPa}[1], E_{0}=860 \mathrm{kV} / \mathrm{cm} / \mathrm{MPa}$ is the breakdown field strength for $\mathrm{SF}_{6}$ gas up to $0.5 \mathrm{MPa}$ [14], $\eta=E_{\text {mean }} / E_{\max }=0.5 \div 0.8$ is the electric field utilization factor (given by $E_{\text {mean }} / E_{\max }$, which are mean and maximum values of the electric field between the contacts, respectively) [13], and $p$ is the $\mathrm{SF}_{6}$ gas pressure.

Assuming the gas pressure of $p=0.45 \mathrm{MPa}$, the Toepler coefficient, as interpolated from the values given above, is $43.3 \mathrm{kV} \cdot \mathrm{ns} / \mathrm{cm}$. For moderate inhomogeneity of the electric field, the value of $\eta=0.7$ can be assumed. Putting those values in (1), the time duration of the spark ignition is $t_{\mathrm{B}}=2 \mathrm{~ns}$, which is in agreement with e.g. [14], where the $t_{\mathrm{B}}$ is reported within the range of $2 \div 20$ ns (depending on the gap distance, the gas pressure and field inhomogeneity).

As the $\mathrm{SF}_{6}$ gas pressure is the GIS design specific factor, and in some cases can also vary with time, for the type test procedures it is not feasible to establish the standardized voltage surges, as it is the case for the tests related to lightning and switching overvoltages. In the case of VFTO testing (including standardized type testing procedures), the operations of real disconnectors are used to generate disconnector design-specific VFTO, instead of using the externally sourced voltage surges which are not practical to be generated.

\subsection{Spark (arc) duration and current switching capability.} After the spark ignition, the capacitive current starts to flow through the spark until it falls below 1-5 A [1]. In order to approximate the spark duration, an exponentially decaying current can be assumed. In the case when the initial value of the current is $10 \mathrm{kA}$ (as it is estimated in [1]) and the characteristic time 
constant is approximately $\tau=7 \mu \mathrm{s}$, the spark is extinguished after the time $t_{\mathrm{ex}}$ given by the following formula:

$$
t_{\mathrm{ex}}=\tau \ln \left(\frac{1 \mathrm{~A}}{10 \mathrm{kA}}\right)=64 \mu \mathrm{s} .
$$

This value is in agreement with the optical observations of sparks, based on which the spark duration of 50-100 $\mu$ s was reported in [1] (see Fig. 3). Spark duration can be further assumed as having a statistical distribution, e.g. the Gaussian one, with a given mean $\mu$ and standard deviation $\sigma$ values.

The spark is extinguished after the time given by the spark duration and after the time instance when the current flowing through the spark decreases below the disconnector capacitive current switching capability [1], which can be assumed as e.g. $1 \mathrm{~A}[1]$.

3.3. Thermal memory of disconnector contacts in SF6. As it is shown in Fig. 3, the subsequent sparks occur in different channels. Thus, it can be assumed that the subsequent sparks do not exhibit thermal memory from the preceding ones, and thus cold breakdowns can be assumed as occurring in thermally not pre-loaded gas conditions [1].
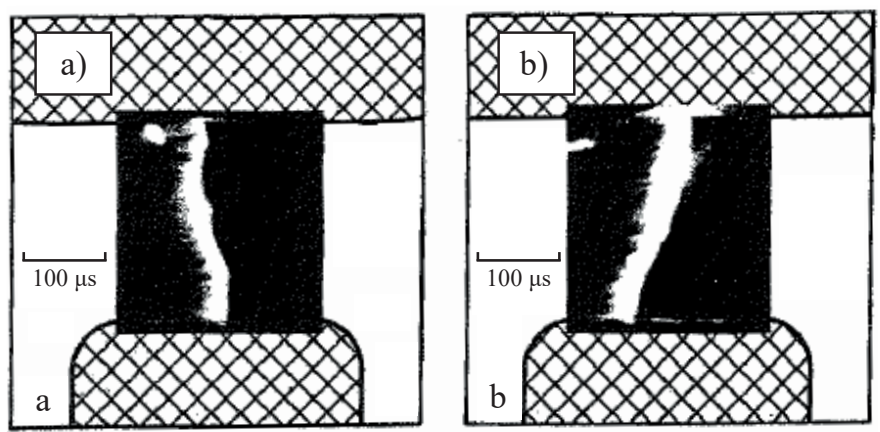

Fig. 3. Optical measurements of subsequent sparks during disconnector operation [1]; the "b" picture is taken approximately $10 \mathrm{~ms}$ after the "a" picture

3.4. Arc and contact gap resistance. The arc resistance model consists of two parts: the model of the arc itself, and the model of transition of the disconnector gap from its insolating to conducing (arcing) state (and the other way round).

Arc resistance is assumed as a constant value, which is in the range of just a few ohms (e.g. it can be assumed as $0.5 \Omega$, as per [15]). The resistance of the contact gap during the transition process can be given by the exponential functions with the time constant obtained from the Toepler equation (1).

The following formulas can be then used based on $[14,15]$ for modeling the ignition $r_{\mathrm{ig}}^{n}(t)$ and extinction $r_{\mathrm{ex}}^{n}(t)$ process of the $n$-th spark in the multi-spark modeling approach:

$$
\begin{aligned}
& r_{\text {ig }}^{n}(t)=r_{\text {arc }}+\left(r_{\text {gap }}-r_{\text {arc }}\right) \cdot e^{-\frac{\left(t-t_{\mathrm{s}}^{n}\right)}{\tau}} \\
& r_{\text {ex }}^{n}(t)=r_{\text {arc }}+\left(r_{\text {gap }}-r_{\text {arc }}\right) \cdot\left(1-e^{-t-\left[t-\left(t_{\mathrm{s}}^{n}-\Delta t_{\mathrm{s}}^{n}\right)\right] / \tau}\right)
\end{aligned}
$$

where $r_{\text {arc }}$ is the arc resistance, $r_{\text {gap }}$ represents the resistance of the open disconnector gap, $\Delta t_{\mathrm{s}}^{n}$ is the time instance of the $n$-th spark ignition, $\Delta t_{\mathrm{s}}^{n}$ is the time duration of the $n$-th spark, and $\tau$ is the time constant according to the Toepler equation (1).

Assuming the exponential character of spark resistance according to (3), the time constant $\tau$ in (3) can be calculated according to the Toepler equation (1), as $\tau=t_{\mathrm{B}} / 3=0.67 \mathrm{~ns}$. This value is in agreement with $[14,15]$, where $\tau=1$ is reported.

The same formulas (in principle) as those given by (3) are being used for VFTO modeling with the single-spark approach (as reported e.g. in $[14,15]$ ) and with the multi-spark approach (as reported in this paper). In the multi-spark approach, however, the spark model given by the formulas (3) is being used for every spark that occurs during the entire operation of the disconnector according to the voltage conditions on the disconnector source- and load- sides $\left(u_{\mathrm{L}}\right.$ and $u_{\mathrm{S}}$ as per Fig. 2, respectively), and according to the relation of the $u_{\mathrm{L}}$ and $u_{\mathrm{S}}$ voltages to the breakdown voltage characteristics.

3.5. Trapped charge and trapped charge voltage. After extinction of each of the sparks, the trapped charge remains on the unloaded capacitive busbar at the disconnector load-side. Voltage $u_{\mathrm{TCV}}$ associated with this trapped charge contributes, jointly with the alternating source-side voltage $u_{\mathrm{S}}$, to the initial conditions for ignition of the next spark. This process is reflected in a step-wise patterned waveform, which is a common pattern for the VFT process as shown in Fig. 2.

The voltage remaining on the disconnector load-side $u_{\mathrm{L}}$ after the opening operation is completed is called trapped charge voltage ( $T C V)$. This $T C V$ contributes to the initial conditions for the first spark ignition in the following closing operation. Due to the slow speed of the disconnector moving contact, the slope of the dielectric strength during the closing operation decreases at a low ratio as compared to the alteration of the $50 / 60 \mathrm{~Hz}$ source-side voltage $u_{\mathrm{S}}$. This causes the first spark during the closing operation most likely to occur at the time instance when source-side voltage $u_{\mathrm{S}}$ reaches its maximum (amplitude) value. During the closing operation, as $B D V$ decreases with the disconnector operating time, subsequent sparks are initiated with lower voltage across the contacts than it is the case for the preceding sparks. In the worst-case scenario of $T C V$ equal -1 p.u., the first spark during the closing operation generates the most severe conditions for VFTO generation. Thus, the first spark during the closing operation is often used for defining the worst-case scenario for VFTO calculation with the use of the single-spark modeling approach, and also when type testing is involved.

3.6. Charge decay. The trapped charge is subject to discharging processes with the time constants related to the disconnector load-side busbar capacitance, to the current leakage conditions and to the polarization currents. Current leakage can take place through and across the spacers (which is a very slow process, taking hours or even days) or through levitating particles. The particles motion under DC conditions can be more severe than that for AC, and may lead to scattering of particles onto insulation surfaces. Such particle motion can lead to appreciable DC 
currents in the $\mu \mathrm{A}$ range, which might discharge the floating section in a relatively short time.

Yet another aspect linked with charge decay is the low frequency cutoff of the external measuring system connected to the disconnector load-side busbar. The voltage measuring system can be represented as a capacitive divider with oscilloscope impedance connected in parallel to the low voltage capacitor (see e.g. $[17,18])$. The equivalent circuit parameters of the measuring system are selected so as to ensure the transfer function in the frequency domain according to the VFTO spectra. The measuring sensor, together with the input impedance of the oscilloscope, contribute to the trapped charge decay with a time constant of equivalent $R C$ elements, which can be in the order of tens of milliseconds. This effect is observed in the measured VFT waveforms as reported in [18].

3.7. Internal damping of VFTO. The internal damping of VFTO waveforms is determined mostly by the spark resistance. The skin effects and leakage currents of the GIS components have a minor effect on VFTO damping, and can thus be neglected [14]. External VFTO are dampened by couplings at the transition points between the GIS and the air-insulated switchgear (AIS) overhead and cable lines.

\section{Breakdown voltage characteristics}

Breakdown voltage describes the dielectric strength of the disconnector contact system, as a function of the disconnector contact gap length. The breakdown voltage (BDV) characteristics is a time-variant function of dielectric contact strength, given as a function of time for the disconnector closing and opening operations.

This section presents two approaches on modeling BDV of the GIS disconnector that can be employed for multi-spark VFTO modeling and analyses. The simplified approach employs liner voltage curve that can be obtained based on the disconnector nameplate data. The full approach requires measurement data obtained from full-scale testing.

For the simplified approach to BDV modeling, it can be assumed that dielectric strength of the disconnector contact gap is a linear function of the gap length (see Fig. 4), and that the contact gap length is a linear function of time (see Fig. 5). The latter can be checked with the measured travel curves of the disconnector no-load operation, as shown in Fig. 5. The maximum value of breakdown voltage equals the rated power frequency withstand voltage $U_{\mathrm{w}}$ across the disconnector insolating distance $s$, where $U_{\mathrm{w}}$ is the equipment nameplate's rated voltage and the value of $s$ is provided by the manufacturer.

With these assumptions, the simplified BDV can be described with the following formulas:

$$
\begin{array}{ll}
\text { opening: } & u_{\mathrm{BDV}}^{\text {opening }}(\mathrm{t})=\frac{U_{\mathrm{w}}}{t_{\text {opening }}}\left(t-t_{0}^{\text {opening }}\right) \\
\text { closing: } & u_{\mathrm{BDV}}^{\text {closing }}(\mathrm{t})=-\frac{U_{\mathrm{w}}}{t_{\text {closing }}}\left(t-t_{0}^{\text {closing }}\right)+U_{\mathrm{w}},
\end{array}
$$

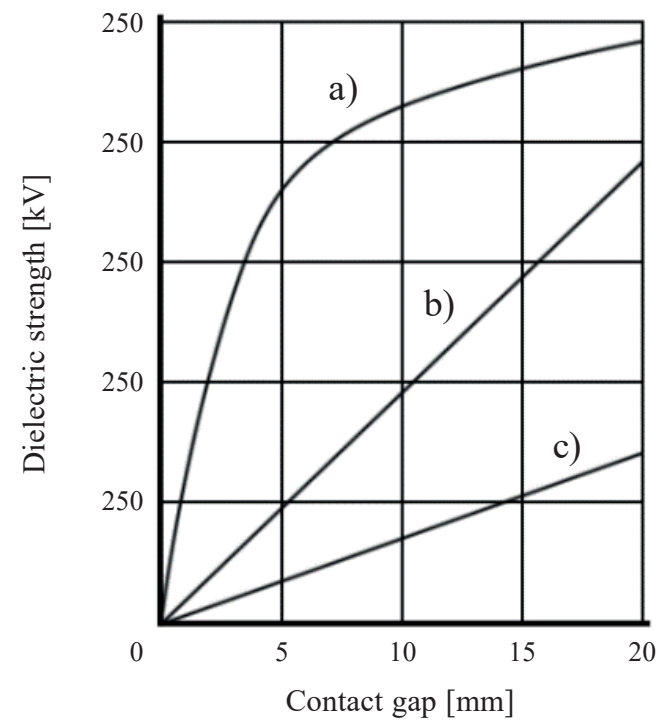

Fig. 4. Dielectric strength of different insulating media as a function of contact gap length: a) high vacuum, b) $\mathrm{SF}_{6}$ at $0.1 \mathrm{MPa}$ pressure, c) air at $0.1 \mathrm{MPa}$ pressure [19]

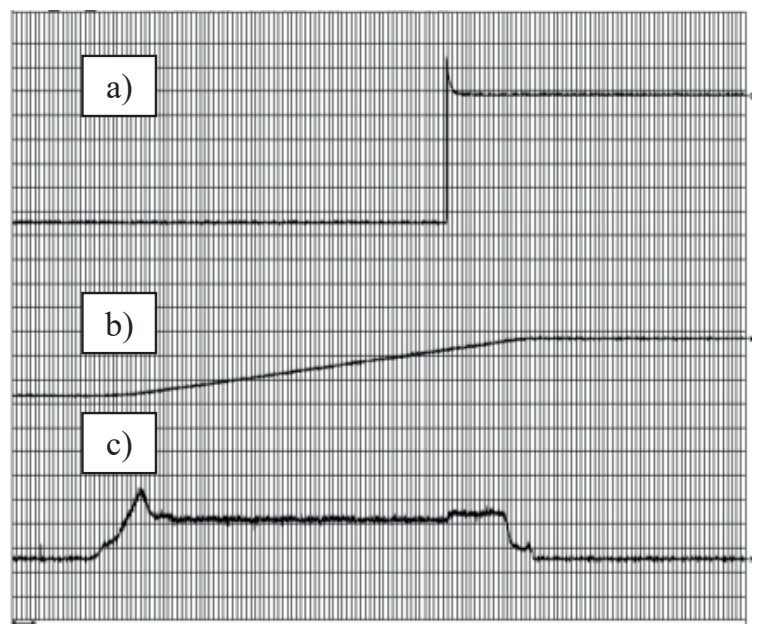

Fig. 5. No-load operation of the disconnector, measured during disconnector type testing: a) contact separation, b) contact distance, c) motor current of the disconnector propulsion drive

where: $u_{\mathrm{BDV}}^{\text {opening/closing }}(t)$ describes instantaneous values of breakdown voltage for the opening- and closing- operation, respectively, $t_{\text {opening/closing }}$ are the opening and closing times, respectively (as provided by the manufacturer or as obtained in no-load operation tests as shown in Fig. 5), $t_{0}^{\text {opening/closing }}$ are the time instances when the disconnector opening or closing operation starts, and $U_{\mathrm{w}}$ is the rated power frequency withstand voltage across isolating distance.

Full approach to VFTO modeling involves multi-spark simulations with design-specific breakdown voltage (BDV) characteristics, as reported in [4]. BDV can be obtained from full-scale measurements of VFTO waveforms as originating from the operations of a real GIS disconnector. For this purpose, the measurement procedures as recommended by the IEC Std. 
[20] for type testing can be used, however other test set-ups that are established as a part of development tests can be utilized as well [4]. Also, VFTO waveforms can be used as obtained from the measuring campaigns that are executed in the test set-ups according to customer specifications, as reported in e.g. [17].

For the specific design of the disconnector contact system, dielectric strength depends on actual length of the disconnector contact gap. For maximum possible voltage across the disconnector contact gap (i.e. for $\left|u_{\mathrm{S}}-u_{\mathrm{L}}\right| \leq 2.1$ p.u., provided that the source-side voltage $u_{\mathrm{S}}$ of $1.1 \mathrm{p}$.u. is assumed as per [20]), the maximum arcing time is in the order of $0.49 \mathrm{~s}$ for the actual BDV of a $1100 \mathrm{kV}$ disconnector [4]. Due to asymmetry of the disconnector contact system, dielectric strength depends not only on the absolute value of voltage across the contact gap $\left(\left|u_{\mathrm{S}}-u_{\mathrm{L}}\right|\right)$ that occurs at the time of spark ignition, but also on the relative values of voltages $u_{\mathrm{S}}$ and $u_{\mathrm{L}}$ (see e.g. Spark 1 and Spark 2 as indicated in Fig. 2).

\section{Multi-spark modeling of VFTO in GIS}

The GIS model for VFTO analyses includes a model of the GIS disconnector, with all of the physical phenomena modeled as described in Section III and Section IV, as well as models of the remaining GIS components and substation equipment.

5.1. Disconnector model. The concept of modeling the GIS disconnector is based on the method initially proposed in [21] and [22] for modeling vacuum circuit breakers by means of the lumped equivalent electrical circuit (LEEC) approach. The chief principle behind the concept (see Fig. 6) is to control nonlinear, time dependent resistance, which is calculated in each step of the simulation process according to formulas (3). The control process is based on calculation of the source- and load-side voltages, $u_{\mathrm{S}}$ and $u_{\mathrm{L}}$, respectively, and comparing the voltages instantaneous values with the design-specific breakdown voltage characteristic of the GIS disconnector [23].

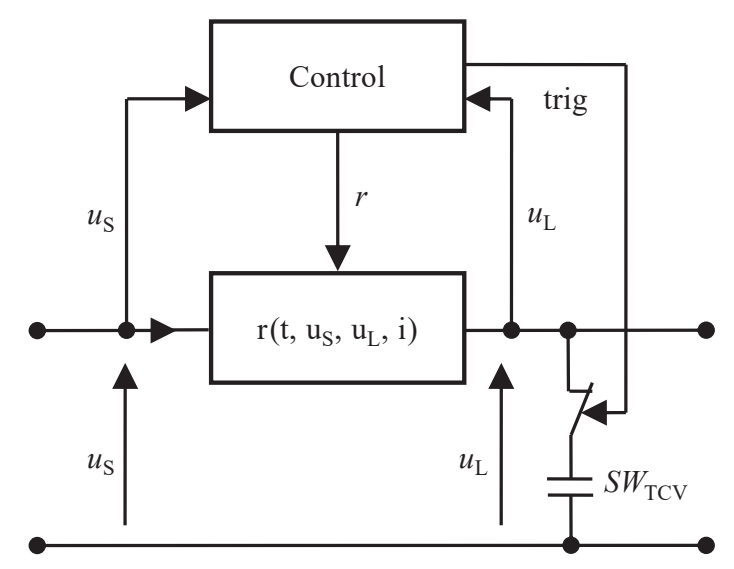

Fig. 6. Illustration of the principle concept of the GIS disconnector model $[12,23] ; r$-EMTP-ATP TACS controlled resistance, $i$ - current flowing through the disconnector, $u_{\mathrm{S}}-$ disconnector source-side voltage, $u_{\mathrm{L}}$ - disconnector load-side voltage, $\mathrm{SW}_{\mathrm{TCV}}$ - triggering switch
The trapped charge voltage is modeled with a lumped capacitor connected to the load-side of the disconnector model, with the initial voltage reflecting the assumed TCV remaining on the disconnector load-side busbar after the completed disconnector opening operation. The capacitor is connected to the circuit by means of an ideal switch, which is triggered so that the trapped charge is connected (switch closed) only before occurrence of the first strike during the disconnector closing operation, and thus the trapped charge does not influence voltage conditions during the remaining stages of the disconnector operation process.

For modeling the entire VFTO process with the multi-spark approach, it is suitable to employ the LEEC modeling approach presented herein. For modeling VFTO with the single-spark approach, both LEEC and full-Maxwell approaches can be used. The method of performing VFTO single-spark simulations with full-Maxwell (e.g. FEM-based) approach is reported in [24, 25].

5.2. Modeling of other power station components. Power station (or test set-up) components other than the operated disconnector are modeled primarily as distributed transmission lines and lumped capacitor elements according to $[14,15]$. The distributed transmission lines are defined by surge impedances and electromagnetic wave travelling times. The equivalent lumped capacitance values are applicable for modeling insulators, GIS busbars and line bushings. Also, switches in open and close positions are modeled with lumped capacitances.

The lumped parameters of the short GIS coaxial busbars can be calculated with the use of standard formulas of capacitances and inductances of coaxial-type conductors. The lumped parameters of components with complex geometry can be derived from full-Maxwell simulations, with e.g. the finite element method as reported in [24]. The full-Maxwell simulations are being performed for specific components' design by using the manufacturer's CAD drawings for different types of equipment.

\section{Multi-spark simulations of VFTO in GIS}

This section presents three cases of VFTO simulations performed with the multi-spark VFTO modeling approach as described in this paper.

A simplified GIS model is first used with the purpose of presenting details of the VFTO generation process in a comprehensive and illustrative manner. Then, a simplified GIS-AIS power station is modeled to compare the multi-spark approach presented herein with the single-spark approach investigated for the same set-up in earlier works $[1,2]$. Initial simulation results presented here for the GIS-AIS simplified station are described in [12]. Examples of multi-spark VFTO simulation results are finally referenced from $[4,6,11,18]$, as performed for the $1100 \mathrm{kV}$ GIS development works with the multi-spark VFTO modeling approach presented herein.

6.1. Illustrative example of the VFTO generation process. Fig. 7a shows an illustrative example of the VFT generation process, simulated for the disconnector opening operation 

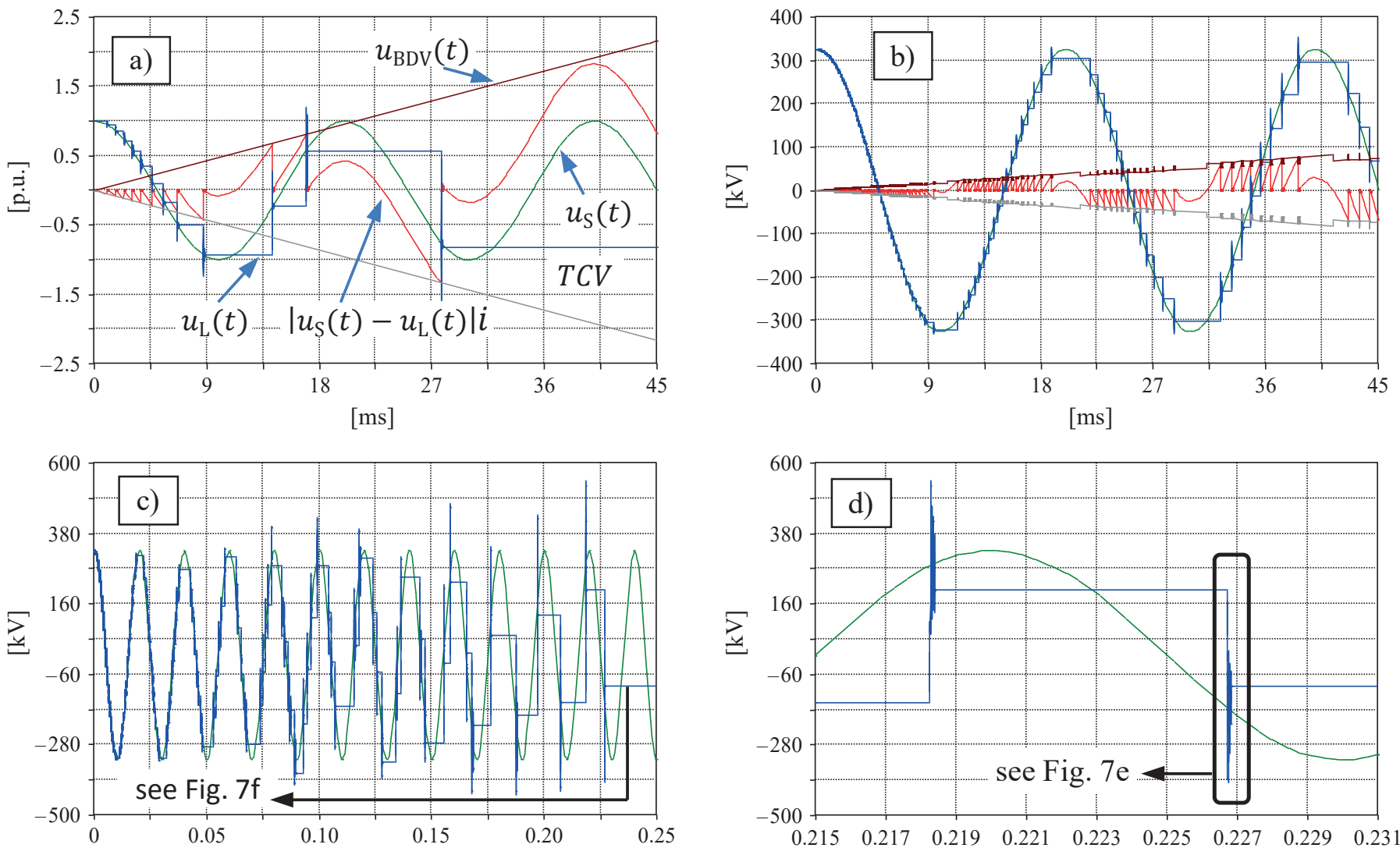

$[\mathrm{s}]$

$[\mathrm{s}]$
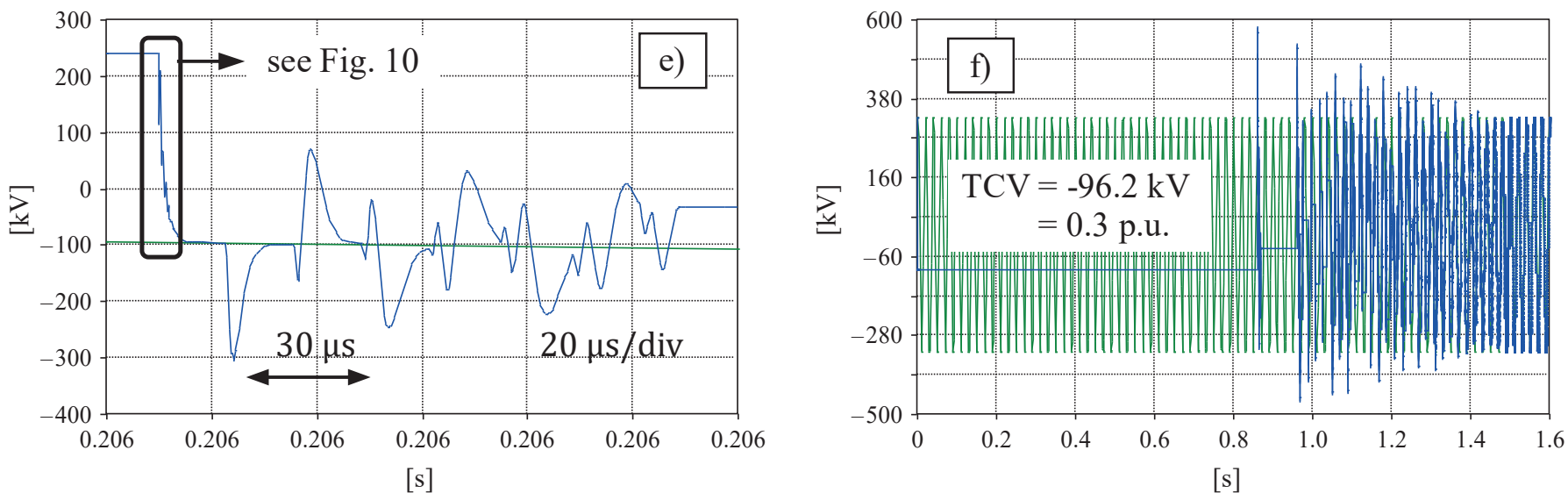

Fig. 7. Representative VFT waveforms for GIS disconnector operations: $u_{\mathrm{S}}-$ source-side voltage (green color), $u_{\mathrm{L}}-$ load-side voltage (blue color), $\left(u_{\mathrm{S}}-u_{\mathrm{L}}\right)$ - voltage drop across disconnector contact system (red color), $u_{\mathrm{BDV}}$ - breakdown voltage (upper/brown color for positive $\mathrm{BDV}$, lower/gray color for negative BDV); a) illustrative waveforms for opening with BDV not related to any actual design; $b-f$ ) waveforms for: opening (b-e) and closing (f) with BDV according to (4); b) first stages of the opening operation, c) entire opening operation, d) last two sparks from the opening operation shown in (c), e) first spark from those sparks shown in (d), f) closing operation for the trapped charge voltage (TCV) value according to (c)

with the multi-spark GIS disconnector model shown in Fig. 6. Voltage across the disconnector contact gap $\left|u_{\mathrm{S}}-u_{\mathrm{L}}\right|$ is being compared with the instantaneous values of breakdown voltage (BDV) characteristics, which varies in time as defined by the $u_{\mathrm{BDV}}$ voltage curves (see Fig. 7a). The resistance used for modeling the spark, as given by formulas (3), is triggered (spark ignition) when voltage across the contact system exceeds breakdown voltage: $\left|u_{\mathrm{S}}-u_{\mathrm{L}}\right| \geq u_{\mathrm{BDV}}$. Voltages $u_{\mathrm{S}}$ and $u_{\mathrm{L}}$ are thus the interfaces between the disconnector model and the external circuit (i.e. the GIS substation and other power system components). The trapped charge voltage (TCV) occurring on the disconnector load-side before the first spark ignition for the closing operation is controlled by the auxiliary switch $S W_{\mathrm{TCV}}$ as shown in Fig. 6.

Figure $7(\mathrm{a}-\mathrm{b})$ shows source- and load-side voltages, $u_{\mathrm{S}}$ and $u_{\mathrm{L}}$, respectively, together with voltage across contacts $\left|u_{\mathrm{S}}-u_{\mathrm{L}}\right|$, 
calculated for the simplified breakdown voltage characteristics according to (4). These figures illustrate how contact voltage $\left|u_{\mathrm{S}}-u_{\mathrm{L}}\right|$ compares with breakdown voltage $u_{\mathrm{BDV}}$ for controlling the spark ignition process. Figure $7 \mathrm{a}$ illustrates the VFT process for unrealistic value of rated power frequency withstand voltage $U_{\mathrm{w}}$ that was assumed for illustration purposes. Figure $7 \mathrm{~b}$ shows the initial part of the opening operation process for realistic value of rated power frequency withstand voltage across the disconnector open contacts $U_{\mathrm{w}}=815 \mathrm{kV}$ (as relevant for $420 \mathrm{kV}$ GIS).

\subsection{VFTO simulations for the simplified GIS-AIS power sta-} tion model. Figure 8 shows a simplified GIS-AIS power station set-up, as proposed and analyzed in early works [1, 2], where the single-spark approach was employed for VFTO modeling.

In the system shown in Fig. 8, an unloaded $66 \mathrm{~m}$ long section of the $420 \mathrm{kV}$ GIS is disconnected from an overhead line of $2000 \mathrm{~m}$ in length. The following models and data were assumed in $[1,2]$ for analyzing the system shown in Fig. 8 with the single-spark VFTO modeling approach. For the GIS bus-duct and for the overhead line, a distributed transmission line was modeled in $[1,2]$ as a single section line. The surge impedance of the GIS bus-duct and of the overhead line were assumed in $[1,2]$ as $Z_{1}=75 \Omega$ and $Z_{2}=320 \Omega$, respectively. The travelling wave speed was assumed in $[1,2]$ as $2.9 \cdot 10^{8} \mathrm{~m} / \mathrm{s}$ and $3.0 \cdot 10^{8} \mathrm{~m} / \mathrm{s}$ for the GIS bus-duct and for the overhead line, respectively. The bushing between GIS and air-insulated switchgear (AIS) was represented in $[1,2]$ by a lumped capacitance of $C_{\mathrm{B}}=500 \mathrm{pF}$.

Figure 9 shows implementation of the system set-up from Fig. 8 in EMTP-ATP simulation software [26], with the multispark VFTO modeling approach presented herein. For implementing the multi-spark approach in the model shown in Fig. 9 [12], linear $B D V$ was assumed, according to (4), with rated power frequency withstand voltage across the disconnector open contacts of $U_{\mathrm{w}}=815 \mathrm{kV}$ (as for the $420 \mathrm{kV}$ rated voltage GIS). The disconnector opening and closing times were assumed as $0.5 \mathrm{~s}$ and $1.6 \mathrm{~s}$, respectively.

Simulation results are shown in Fig. 7(b-f), where the source-side $u_{\mathrm{S}}$ and load-side $u_{\mathrm{L}}$ voltages are marked in green and blue colors, respectively. Fig. 7(b-e) show the opening op-

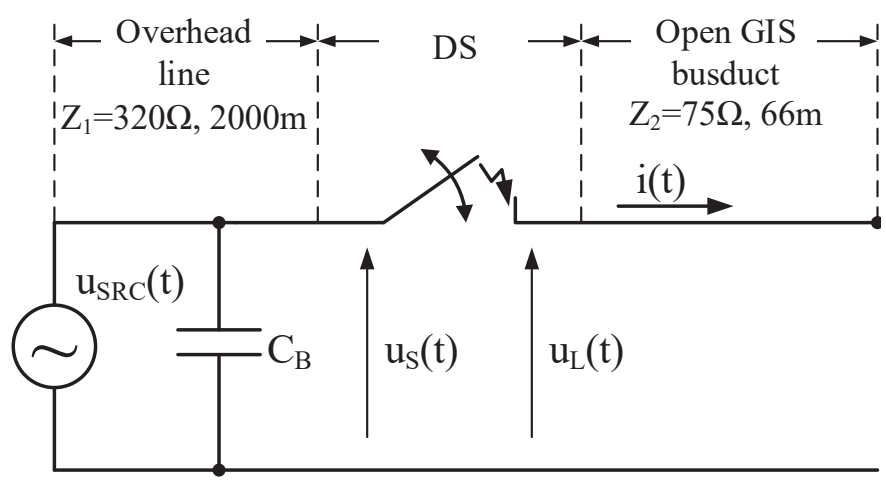

Fig. 8. Simplified GIS-AIS power station set-up, as proposed and analyzed with the single-spark VFTO modeling approach in $[1,2]$

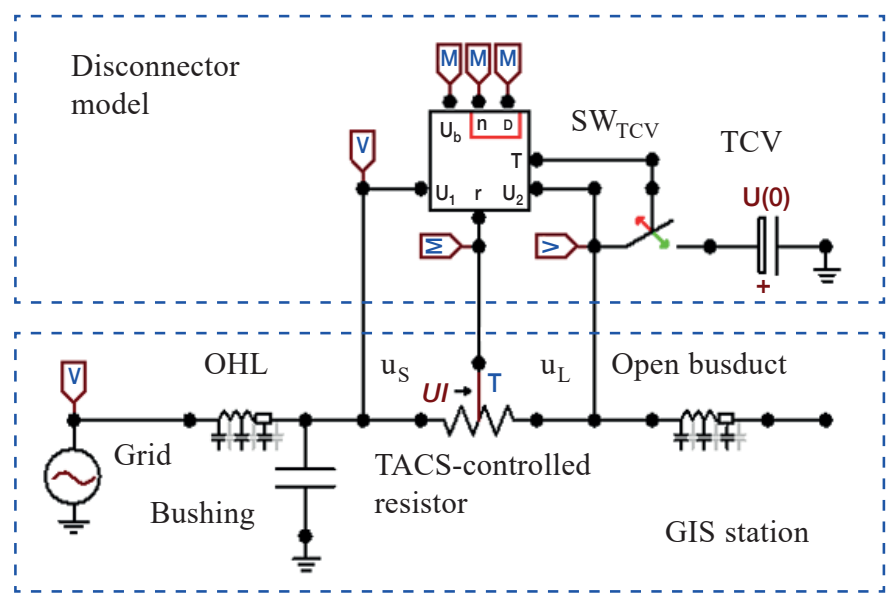

Fig. 9. Implementation of the set-up from Fig. 8 [12] in EMTP-ATP simulation software [26]

eration, while Fig. 7f shows the closing operation of the disconnector model shown in Fig. 9. For the opening operation, the total number of sparks is 153 , with 12 sparks leading to VFTO with peak values exceeding $1.2 \mathrm{p}$.u. The trapped charge voltage $(T C V)$ is $-96.2 \mathrm{kV}$ (i.e. -0.2 p.u.), as indicated in Fig. 7c for the opening operation. This $T C V$ value is in agreement with the value reported in [2]. It was also used as an initial voltage condition for the subsequent closing operation, as shown in Fig. $7 \mathrm{f}$. For the closing operation shown in Fig. 7f, the total number of sparks is 470, with 21 sparks leading to VFTO with peak values exceeding 1.2 p.u. VFTO with significant peak values are clearly seen in Fig. 7c and Fig. 7f for the opening and closing operations, respectively. Figure $7(\mathrm{~d}-\mathrm{e})$ show zoomed last peaks of the VFTO occurring during the opening operation, as shown in Fig. 7c. In Fig. 7e, the co-relation between the voltage wave shape and the travelling wave transition time for the $2000 \mathrm{~m}$ overhead line is indicated. A part of Fig. 7e is zoomed in Fig. 10 and further discussed below.

It can be seen from Fig. 7c and Fig. 7f that for a significant number of sparks the VFTO of significant peak values are generated. The peaks of VFTO are scattered, with statistical distribution specific to particular parameters of the VFTO generation process (see e.g. $[4,8,9,11]$ ). Also, the trapped charge voltage as well as the arcing time and the number of sparks, strongly depend on the disconnector design. These aspects can be modeled with design specific BDV obtained from full-scale measurements, as reported in [4].

Figure 10 shows a comparison of the VFTO waveforms obtained for a selected spark, as simulated with the multispark approach reported in this paper (see Fig. 10a), and the results obtained with the single-spark approach as reported in $[1,2]$ (see Fig. 10b). Fig. 10a shows one spark selected from the sparks shown in Fig. 7c (see also Fig. 7e for the particular part of the waveform shown in Fig. 10a). The VFTO waveform shown in Fig. 10a is plotted in a time-scale, zoomed-in so it fits the time-scale of the single-spark shown in Fig. 10b. This simplifies comparison of the wave effects observed. The voltage scale in Fig. 10a is in the base unit quantity, so it is consistent 

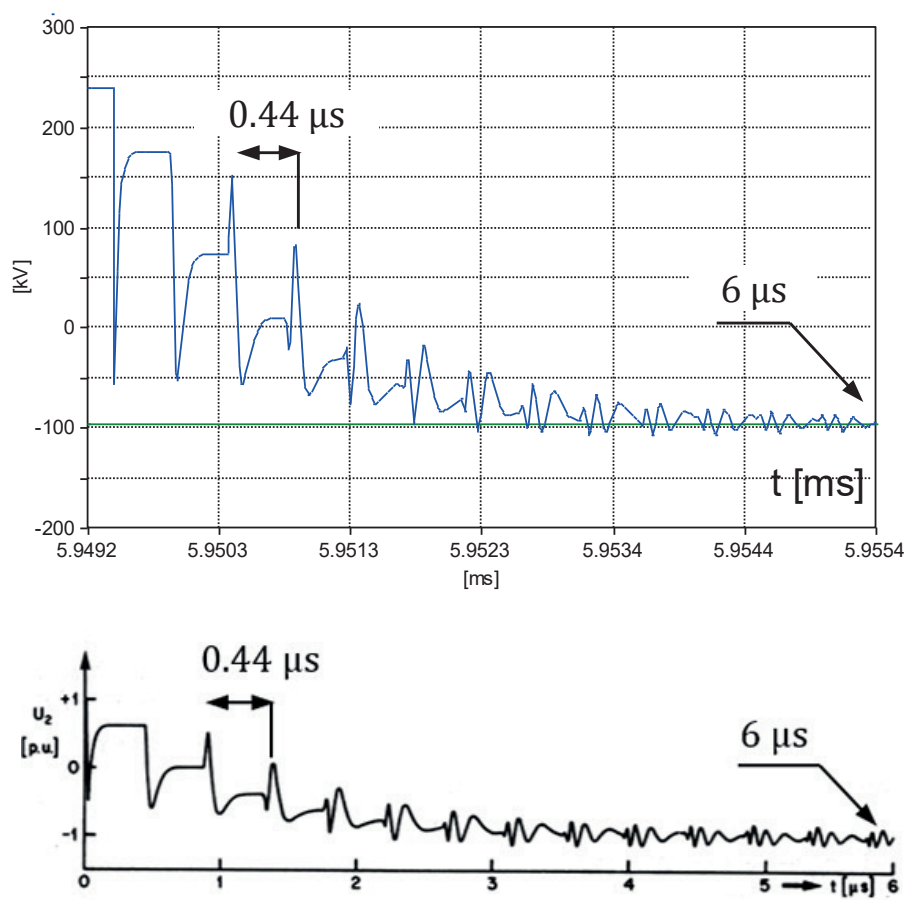

Fig. 10. Load-side voltage $u_{\mathrm{L}}$ [12]: a) simulated with the use of the multi-spark approach as presented in this paper (one spark zoomed-in from Fig. 7c, as indicated in Fig. 7e), b) calculated with the use of the single-spark approach as reported in $[1,2]$

with Fig. 7e, which Fig. 10a originates from. The voltage scale in Fig. 10b is in the per unit quantity, originally used in works $[1,2]$, which Fig. 10b originates from. The co-relation between the voltage wave shapes and the transmission time in the $60 \mathrm{~m}$ GIS busduct is indicated. As the VFTO generation process is linear, the wave shapes for any of the spark events are linearly dependent on the initial voltage conditions occurring at the time instance of spark ignition. Hence, the wave shape co-relation as seen in Fig. 10 can be observed for any of the sparks that can be selected from the entire disconnector operation process (as shown e.g. in Fig. 7c).

The characteristic times, as indicated in Fig. 7e and Fig. 10(a-b), are related to the travelling wave reflections in the test set-up used for simulations as shown in Fig. 8 and Fig. 9. This is a result of the distribution nature of the components employed. In the simplified model shown in Fig. 8 and Fig. 9, only three points of surge impedance discontinuity exist: at the end of the overhead line, at the open-end of the GIS bus-duct, and at the connection between the two latter elements (i.e. at the disconnector switch). After spark ignition, two electromagnetic waves begin to propagate, originating from voltage flashover in the disconnector contact system. One of the wave reaches the open-end of the $66 \mathrm{~m}$ bus-duct and then it is reflected. After twice the travelling time, the wave reaches the disconnector. The $0.44 \mu$ s travelling time related to the $66 \mathrm{~m}$ bus-duct is depicted in Fig. 10(a-b). Another wave propagates in the opposite direction, reaches the end of the $2000 \mathrm{~m}$ overhead line and then runs back in $27 \mu$ s of travelling time. This time is slightly delayed by bushing capacitance $C_{\mathrm{B}}$, i.e. the connection between the GIS disconnector and the AIS overhead line. The time of $15 \mu \mathrm{s}$, as related to the latter travelling wave, is indicated in Fig. 7e.

As it can be seen in Fig. 10, the single- and multi-spark approaches provide the same results as far as a single VFTO waveform (i.e. the VFTO waveform originating from a given spark event) is analyzed. The multi-spark approach, however, offers the very meaningful additional insights on the entire VFTO generation process, and thus can be further used to analyze the VFTO impact on design of the GIS components, for insulation co-ordination studies as well as for selection and design of the VFTO mitigation solution. These aspects are further outlined in the following sections of this paper.

6.3. VFTO simulations for full-scale GIS development. Figure 11 shows three examples of full-scale $1100 \mathrm{kV}$ GIS test
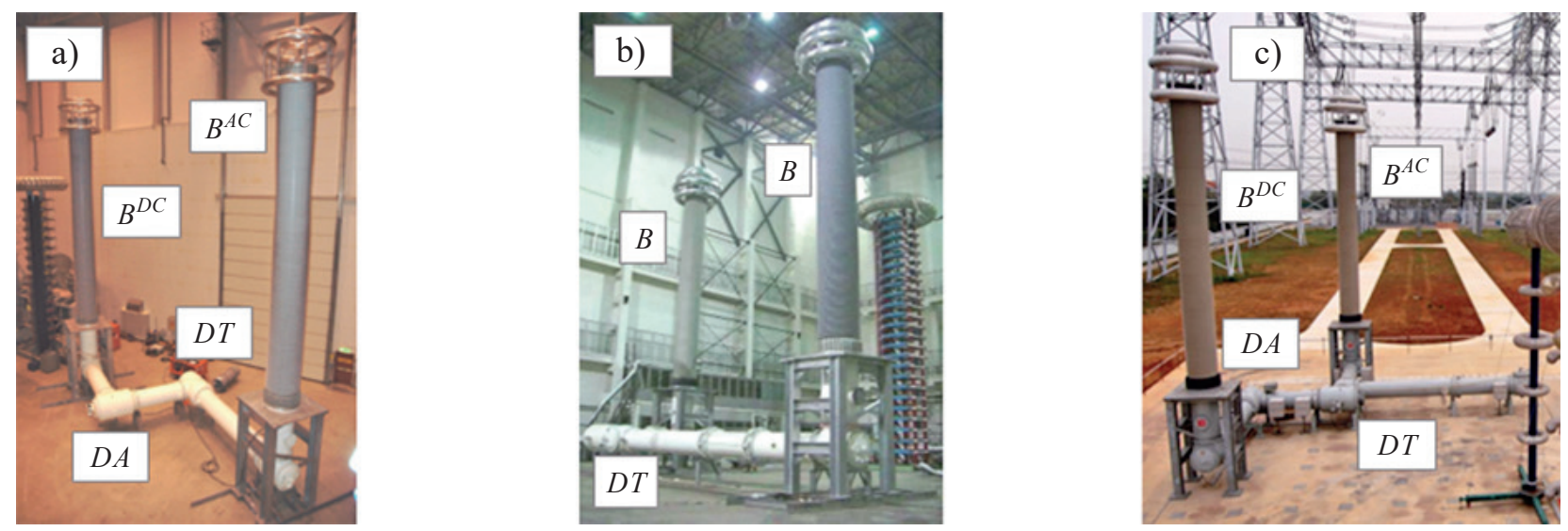

Fig. 11. Full-scale $1100 \mathrm{kV}$ GIS test set-ups: a) test set-up at the STRI HV laboratory in Sweden [10] for type testing of the GIS disconnector according to IEC Std. [20]; b) test set-up according to IEC Std. [20] established for development tests of the $1100 \mathrm{kV}$ GIS disconnector; c) test set-up at the Wuhan $1100 \mathrm{kV}$ GIS station $[17,27]$ for testing of GIS disconnectors of different designs; DT - disconnector under test, DA - auxiliary disconnector, B - GIS-AIS bushings 
set-ups, for which the multi-spark approach to VFTO modeling was employed, as presented in this paper. The test set-up in Fig. 11a was established by ABB at the STRI HV laboratory in Sweden [10] for type testing of the $1100 \mathrm{kV}$ GIS disconnector according to type testing procedures as recommended by IEC Std. [20]. The test set-up shown in Fig. 11b was established by ABB for testing of a new $1100 \mathrm{kV}$ GIS disconnector design at the disconnector development stages [4]. The test set-up shown in Fig. 11c was established by the State Grid Corporation of China (SGCC) in Wuhan's $1100 \mathrm{kV}$ GIS station [17, 27] for research on and testing of $1100 \mathrm{kV}$ GIS disconnectors of different designs.

The test set-ups shown in Fig. 11 served for performing VFTO analyses in several works that have been conducted based on VFTO measurements and simulations, as reported e.g. in $[4,8,9,11,17,18,23,27,28]$. For modeling the entire VFT process, the multi-spark approach as presented in this paper was employed. The simulation results performed with the multispark approach presented herein were reported in several works, e.g. $[4,6,11,18,23,28]$, as well as served as part of the ABB contribution [4] to CIGRE Technical Brochure TB-542 [5].

Reference [18] presents an example of the VFT process measured and simulated for an opening operation of a $1100 \mathrm{kV}$ GIS disconnector, for the test set-up shown in Fig. 11a. Meanwhile, Ref. [4] presents the method of obtaining the breakdown voltage characteristics of the GIS disconnector based on fullscale measurements in the HV test set-up.

The trapped charge voltage (TCV) that remains on the load-side of the disconnector after the disconnector opening operation is completed, is a statistical variable that establishes initial voltage conditions for the subsequent closing operation of the disconnector. To obtain the TCV distributions, the multispark VFTO simulations are being performed for a number of disconnector opening operations, after which the TCV values are recorded. The TCV distributions are dependent on the disconnector design $[6,11]$. This includes dependence on the disconnector moving contact speed and its travelling curve characteristics, $\mathrm{SF}_{6}$ gas characteristics and pressure, and dielectric design of the disconnector contact system. The TCV distributions obtained with the multi-spark VFTO modeling approach can thus serve as a starting point for establishing initial conditions for subsequent design-specific VFTO calculations and for the insulation co-ordination studies.

Example of simulation results of the TCV distributions, obtained with the multi-spark approach presented herein for different operating speeds of the disconnector moving contact for the test set-ups shown in Fig. 11, are reported in [4, 6, 10, 11]. The simulations are performed with the breakdown voltage characteristics (BDV) obtained from the full-scale measurements, as reported in [4]. The TCV cumulative distributions are calculated for different operating speeds of the disconnector moving contact. It is shown in [11] that the probability of high TCV values significantly decreases with the increase of the moving contact speed.

While performing VFTO simulations, such parameters like number of sparks and arcing time can be calculated. Examples of such calculations performed with the multi-spark approach presented herein for the test set-up shown in Fig. 12c are reported in [4].

6.4. VFTO damping for UHV GIS development. For recent development projects of UHV GIS, an evaluation is taking place of whether a VFTO damping solution is required to be applied in the GIS in order to maintain the VFTO peak values at an acceptable level. In some cases the VFTO peak values can be kept within acceptable limits by means of careful design of the GIS insulation systems, in other - application of the VFTO damping solution becomes a necessity. If the VFTO damping solution is required to be applied, selection of the damping method is being evaluated, firstly throughout extensive simulation studies and then by means of measurements performed in development and type test set-ups (see Fig. 11).

Two major types of VFTO damping methods are presently being investigated in both industry and the academia. One branch is based on dissipation of the energy associated with the electromagnetic wave that the VFTO consist of. These methods require insertion to the GIS current path of an energy dissipating element, which can be of resistive [29], magnetic [30, 31] or resistive-inductive [32] type. Also, internal resonators [33] and surge arresters [34] are being proposed to serve this purpose.

Second variety of the VFTO damping methods involves control over the voltage conditions that precede voltage breakdowns during the switching device operation, and thus determine severity of the VFTO generation process. These methods are primarily based on control over TCV distributions for the disconnector opening operation process $[6,10,11]$, for the analyzing of which the multi-spark modeling approach presented herein is needed as described above. Also, the VFTO pattern can be controlled by means of imposing a control algorithm over voltage conditions at the time instances of spark ignitions. This method has been recently reported in [28] as investigated with the multi-spark VFTO modeling approach presented herein. The initial voltage conditions of the travelling waves originating from voltage flashovers in the disconnector contact system can also be controlled by modifying the disconnector contact system design, as reported in [35].

For each of the VFTO damping solutions as outlined above, the multi-spark VFTO modeling approach allows one to assess the need for application of the VFTO damping solution, and then to assess effectiveness of the VFTO damping solution employed, to support the damping solution dimensioning, and to contribute to the final GIS design.

\section{Conclusions}

Modeling of very fast transient overvoltages (VFTO) in gas-insulated switchgear (GIS) is currently being increasingly employed by HV and UHV GIS design and development engineers to support cost-effective design and type testing of GIS components and to ensure reliable operation of GIS power stations. Due to high costs of testing HV and UHV equipment, the comprehensive simulation methods, based on validated simulation models, are being extensively used to support development 
works of GIS components and power stations. Also, insulation coordination studies involve VFTO analyses based on simulation methods at the design and commissioning phases of the GIS power station delivery and operation.

The paper presents the multi-spark approach to VFTO modeling in GIS that allows one to analyze the entire process of VFTO generation, involving in the design work for such design-specific parameters as VFTO distributions, trapped charge voltage distributions, arcing time and the number of sparks. Accurate modeling of the VFTO with the multi-spark approach as employed for a particular disconnector design, is often required to make a decision on the application of the VFTO damping solution, and for supporting the damping solution dimensioning and its optimum design. This is specifically of importance when the reliability and cost of the UHV GIS solutions are of concern.

The multi-spark approach to VFTO modeling in GIS, as presented in this paper, has been extensively used in recent development works of UHV GIS. It has been proven as a suitable approach for any GIS, however, applicable primarily for development and design works, and insulation co-ordination studies, of GIS components and power stations rated at $\mathrm{HV}$ and UHV levels.

\section{REFERENCES}

[1] G. Ecklin and D. Schlicht, "Overvoltages in GIS caused by the operation of isolators", Proc. 1979 of the Brown Boveri Research Centre, Baden, Switzerland, September 3-4, 1979.

[2] S.A. Boggs, F.Y. Chu, N. Fujimoto, A. Krenicky, A. Plessl, and D. Schlicht, "Disconnect switch induced transients and trapped charge in gas-insulated substations", IEEE Trans. Power Apparatus and Systems PAS-101 (10), 1982.

[3] High-Voltage Switchgear and Controlgear - Part 203: Gas-Insulated Metal-enclosed Switchgear for Rated Voltages Above $52 \mathrm{kV}$, IEC Standard 62271-203, Nov. 2003.

[4] M. Szewczyk, M. Kuniewski, W. Piasecki, and M. Florkowski "Determination of breakdown voltage characteristics of 1' $100 \mathrm{kV}$ disconnector for modeling of VFTO in gas-insulated switchgear", IEEE Trans. Power Delivery 31 (5), 2151-2158, Dec. 2015.

[5] Working Group C4.306, Insulation Coordination for UHV AC Systems, CIGRE Technical Brochure TB-542, June 2013.

[6] U. Riechert, M. Bösch, M. Szewczyk, W. Piasecki, J. Smajic, A. Shoory, S. Burow, and S. Tenbohlen, "Mitigation of very fast transient overvoltages in gas insulated UHV substations", in Proc. CIGRE, 2012.

[7] A. Tavakoli, A. Gholami, H. Nouri, and M. Negnevitsky, "Comparison between suppressing approaches of Very Fast Transients in gas-insulated substations (GIS)", IEEE Trans. Power Delivery 28 (1), 303-310, Jan 2013.

[8] Y. Shu, W. Chen, Z. Li, M. Dai, Ch. Li, W. Liu, and X. Yan, "Experimental research on very-fast transient overvoltage in $1100-\mathrm{kV}$ gas-insulated switchgear", IEEE Trans. Power Delivery 28 (1), 458-466, Jan. 2013.

[9] S. Yinbiao, H. Bin, L. Ji-Ming, Ch. Weijiang, B. Liangeng, $\mathrm{X}$. Zutao, and Ch. Guoqiang, "Influence of the switching speed of the disconnector on very fast transient overvoltage", IEEE Trans. Power Delivery 28 (4), 2080-2084, Oct. 2013.

[10] U. Riechert and W. Holaus, "Ultra high-voltage gas-insulated switchgear - a technology milestone", European Transactions on Electrical Power 22 (1), 60-82, May 2011.
[11] M. Szewczyk, W. Piasecki, M. Stosur, U. Riechert, and J. Kostovic, "Impact of disconnector design on very fast transient overvoltages in gas-insulated UHV switchgear", presented at the 17th Int. Symp. High Voltage Eng. (ISH), Hannover, Germany, Aug. 22-26, 2011.

[12] M. Szewczyk, M. Stosur, W. Piasecki, M. Fulczyk, M. Florkowski, M. Steiger, and J. Kostovic, "New disconnector model for very fast transient studies in high voltage gas-insulated substation", in Proc. 2010 European EMTP-ATP Conference (EEUG), Helsinki, Finland, Aug. 6-18, 2010.

[13] CIGRE Working Group SC 33 and 13, "Very fast transient phenomena associated with gas insulated substations", CIGRE Session 1988

[14] D. Povh, H. Schmitt, O. Volcker, R. Witzmann, P. Chewdhuri, A. E. Imece, R. Iravani, J. A. Martinez, A. Keri, and A. Sarshar, "Modeling and analysis guidelines for very fast transients", IEEE Power Engineering Review 17 (13), 1996.

[15] J.A. Martinez, D. Povh, P. Chowdhuri, R. Iravani, and A.J.F. Keri, "Modeling guidelines for very fast transients in gas insulated substations", IEEE PES Special Publication of the IEEE Working Group on Modeling and Analysis of System Transients, 1998.

[16] N. Fujimoto, E.P. Dick, S.A. Boggs, and G.L. Ford, "Transient ground potential rise in gas insulated substations - Experimental studies", IEEE Trans. Power Apparatus and Systems PAS-101 (10), 3603-3609, Oct. 1982.

[17] Y. Gongchang, L. Weidong, Ch. Weijiang, G. Yonggang, and L. Zhibing, "Development of full frequency bandwidth measurement of VFTO in UHV GIS", IEEE Trans. Power Delivery 28 (4, 2550-2557, Oct. 2013.

[18] M. Szewczyk, M. Stosur, W. Piasecki, M. Kuniewski, P. Balcerek, M. Florkowski, and U. Riechert, "Measurements and simulations of very fast transients during disconnector", Przeglad Elektrotechniczny 88 (11B) (2012).

[19] K.C. Agrawal, Industrial Power Engineering and Application Handbook, Newnes Power Engineering series, 2001.

[20] High-Voltage Switchgear and Controlgear-Part 102: Alternating Current Disconnectors and Earthing Switches, IEC 62271-102.

[21] J. Helmer and M. Lindmayer, "Mathematical modeling of the high frequency behaviour of vacuum interrupters and comparison with measured transients in power systems", Proc. 1996 17th International Symposium on Discharges and Electrical Insulation in Vacuum, Berkeley, USA, 1996.

[22] S.M. Wong, L.A. Snider, and E.W.C. Lo, "Overvoltages and reignition behaviour of vacuum circuit breaker", Proc. 2013 International Conference on Power Systems Transients (IPST), New Orleans, USA, 2003.

[23] M. Szewczyk, T. Kuczek, P. Oramus, and W. Piasecki, "Modeling of repetitive ignitions in switching devices: case studies on vacuum circuit breaker and GIS disconnector", Lecture Notes in Electrical Engineering, Volume 324, Analysis and Simulation of Electrical and Computer Systems, Springer Verlag, 2015 (ISBN: 978-3-319-11247-3)

[24] M. Szewczyk, K. Kutorasiński, M. Wroński, M. Florkowski, "Full-Maxwell simulations of very fast transients in GIS: case study to compare 3D and 2D-axisymmetric models of $1100 \mathrm{kV}$ test set-up", IEEE Trans. Power Delivery [accepted for publication, published in IEEEXplore, DOI: 10.1109/ TPWRD.2016.2527823], February 2016.

[25] J. Smajic, W. Holaus, J. Kostovic, and U. Riechert, "3D Full-Maxwell simulations of very fast transients in GIS", IEEE Trans. Magnetics 47 (5), 1514-1517, May 2011. 
[26] H.W. Dommel and W.S. Meyer, "Computation of electromagnetic transients", Proceedings of IEEE 62 (7), 983-993, July 1974.

[27] W. Chen, H. Wang, B. Han, L. Wang, G. Ma, G. Yue, Z. Li, and $\mathrm{H}$. Hu, "Study on influence of disconnector characteristics on very fast transient overvoltage in $1100 \mathrm{kV}$ gas-insulated switchgear", to be published.

[28] M. Szewczyk, M. Kuniewski, "Controlled voltage breakdown in disconnector contact system for VFTO mitigation in gas-insulated switchgear (GIS)", [accepted for publication, published in IEEEXplore, DOI: 10.1109/TPWRD.2017.2676178], March 2017.

[29] Y. Yamagata, K. Tanaka, S. Nishiwaki, N. Takahashi, T. Kokumai, I. Miwa, T. Komukai, and K. Imai, "Suppression of VFT in $1100 \mathrm{kV}$ GIS by adopting resistor-fitted disconnector", IEEE Tran. Power Delivery 1 (2), 872-880, April 1996.

[30] M. Szewczyk, J. Pawłowski, K. Kutorasiński, W. Piasecki, M. Florkowski, and U. Straumann, "High-frequency model of magnetic rings for simulation of VFTO damping in gas-insulated switchgear with full-scale validation", IEEE Trans. Power Delivery 30 (5), 2331-2338, October 2015.
[31] M. Szewczyk, K. Kutorasiński, J. Pawłowski, W. Piasecki, and M. Florkowski, "Advanced modeling of magnetic cores for damping of high-frequency power system transients", IEEE Trans. on Power Delivery 31 (5), 2431-2439, Apr. 2016.

[32] M. Szewczyk, W. Piasecki, M. Stosur, M. Florkowski, U. Riechert, "Damping of VFTO in gas-insulated switchgear by a new coating material", IEEE Trans. Power Delivery 31 (6), 2553-2558, Feb. 2016.

[33] J. Smajic, A. Shoory, S. Burow, W. Holaus, U. Riechert, and S. Tenbohlen, "Simulation-based design of HF resonators for damping very fast transients in GIS", IEEE Trans. Power Delivery, 2014.

[34] S. Burow, U. Straumann, W. Köhler, and S. Tenbohlen, "New methods of damping very fast transient overvoltages in gas-insulated switchgear", IEEE Trans. Power Delivery 29 (5), 23322339, October 2014.

[35] M. Szewczyk, W. Piasecki, M. Wroński, and K. Kutorasiński, "New concept for VFTO attenuation in GIS with modified disconnector contact system", IEEE Trans. Power Delivery 30 (5), 2138-2145, Dec 2014. 